\title{
Research and Application of Automatic Fault Diagnosis and Isolation Device for Distribution Line
}

\author{
Keyin $\mathrm{Jia}^{1}$, Cheng Wang ${ }^{1}$, Shiyang Zheng ${ }^{1}$, Junfeng Liu ${ }^{1}$, Minghongtian Chen ${ }^{1}$ \\ ${ }^{1}$ Siping Power Supply Company, State Grid Jilin Electric Power Company, 136000 Jilin, China
}

\begin{abstract}
This paper takes the equipment of a specific project as an example to develop an automatic fault diagnosis and isolation device for $10 \mathrm{kV}$ overhead line or cable line when a user has grounding and shortcircuit faults, so as to realize fast fault location, isolation and recovery, reduce the power outage time of users, and thus improve the reliability of distribution network. The project takes the $10 \mathrm{kV}$ South and North Trunk Lines 'hand in hand' power supply network as the research object, and studies the solutions of the automatic fault isolation function and the restoration of power supply, hoping to further improve the level of rapid power recovery, reduce power loss, and improve the reliability, automation and information level of power supply for reference.
\end{abstract}

\section{Introduction}

Automatic diagnosis and isolation device for distribution fault is a fault isolation device which integrates high voltage vacuum circuit breaker and intelligent controller. The device has manual and electric operation functions, uses vacuum arc extinguishing and SF6 gas insulation medium is used. The circuit breaker can also be combined with an isolating switch and can be equipped with an intelligent controller. As a power supply device, the selfprovided PT becomes an only circuit breaker, which has the output function of voltage and current signal, and also has the characteristics of controllability. The device not only can operate independently as a complete system, but also can be extended compatible with the power automation SCADA system.

\section{Principle of automatic line fault diagnosis and isolation device}

Automatic diagnosis and isolation device for distribution fault is a fault isolation device which integrates high voltage vacuum circuit breaker and intelligent controller. The device has manual and electric operation functions, uses vacuum arc extinguishing and SF6 gas insulation medium is used. The circuit breaker can also be combined with an isolating switch and can be equipped with an intelligent controller. As a power supply device, the selfprovided PT becomes an only circuit breaker, which has the output function of voltage and current signal, and also has the characteristics of controllability. The device not only can operate independently as a complete system, but also can be extended compatible with the power automation SCADA system. With the function of fault location and intelligent fault indicator matching, it can detect a series of situations on-line quickly and accurately, for example, short circuit fault, grounding fault, power transmission and so on. At the same time, it can collect the characteristic information of the fault and the waveform information when the fault occurs, and then send it to the monitoring center. The fault software can be used to realize alarm display notification, data analysis, cloud computing, report query and fault retrieval statistics, which can guide maintenance personnel to locate fault points quickly and accurately, and then improve work efficiency and reduce work intensity.

\section{System integration module of the device}

\subsection{Integrated system of fault isolation device and fault location device}

Each module is assembled and integrated to form a complete set of automatic fault diagnosis and isolation device. The system consists of front-end monitoring system, transmission network and background system. The overall block diagram is shown in Fig.1.

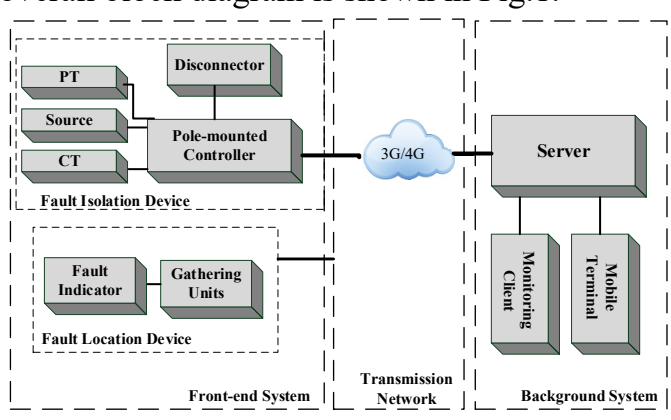

Fig.1 Overall block diagram of integrated system 
Front-end system consists of fault isolation device and fault location device. Separately, fault isolation device consists of controller, voltage transformer, current transformer, $10 \mathrm{kV}$ power source and disconnector and fault location device is made up with fault indicator and gathering units. The transmission network is wireless $3 \mathrm{G}$ or $4 \mathrm{G}$ network. Besides, the background system is a server platform which manages front-end data information.

With the help of the client, the detection center can realize the real-time observation of each control point and the field situation. Recurring to a series of data sent by the scene, such as current, voltage and related fault status, monitoring and maintenance personnel can compare and analyze them. If there is abnormal warning information, targeted emergency treatment should be immediately started, so that the safe operation of high-voltage lines can be effectively guaranteed.

\subsection{Automatic fault diagnosis module}

Automatic fault diagnosis is realized by signal acquisition, fixed value storage, action signal output and so on. This project is aiming at the grounding and short circuit fault of a user of $10 \mathrm{kV}$ overhead line or cable line, develops an automatic fault diagnosis and isolation device to realize the rapid location, isolation and recovery of the fault, to reduce the power outage time of the users, and thus improves the reliability of the distribution network.

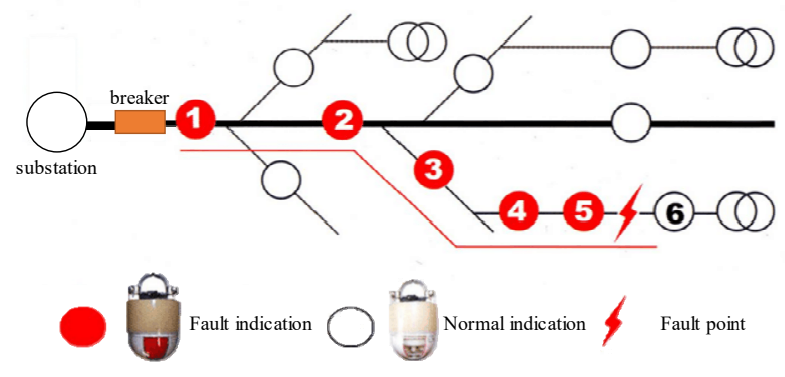

Fig.2 Line fault location diagram

The fault information can be sent to the mobile phones of the person in charge of the power supply production department and the line maintenance personnel by SMS. The judgment and location of line fault is shown in Fig.2.

\section{$3.310 \mathrm{kV}$ high voltage energy acquisition unit}

By taking energy units from $10 \mathrm{kV}$ side to supply the system with power, high voltage electric power acquisition consists of two parts. The one is supplying electricity to isolation device through PT, the other is fault indicator sensor power unit supplied by CT. The power supply problem of the system is solved by high voltage energy acquisition.

\subsection{Fault isolation module}

Local fault isolation is realized by utilizing the driving circuit to control the on-off of the $10 \mathrm{kV}$ circuit breakers. The fault isolation device, which systematically integrates high voltage vacuum circuit breaker and intelligent controller, has the functions of manual and electric operation. The device uses vacuum arc extinguishing and SF6 gas as insulation medium. The circuit breaker can also form a combined circuit breaker with an isolating switch. It can be equipped with intelligent controller and selfprovided PT as power supply. It becomes an intelligent circuit breaker with voltage and current signal output and can be controlled.

\section{Application of automatic line fault diagnosis and isolation device system}

\subsection{Application of automatic fault isolation system}

The automatic distribution line fault diagnosis and isolation device, which integrates high voltage vacuum circuit breaker and intelligent controller, has the functions of manual and electric operation. The device uses vacuum arc extinguishing and SF6 gas as insulation medium. The circuit breaker can also form a combined circuit breaker with an isolating switch. It can be equipped with intelligent controller and self-provided PT as power supply. It becomes an intelligent circuit breaker with voltage and current signal output and can be controlled.

1. Automatic isolation of interphase short circuit faults in user areas

In the event of interphase short circuit fault on the user branch line, the fault isolator shall open and lock immediately after the protection tripping of the substation circuit breaker or reclose. After the substation recovers, the other users on the feeder quickly restore power supply.

2. Rapid location of fault points

The fault of the user branch line causes the protection action of the boundary switch. After the blackout occurs, the responsible user can report the situation actively, so that the power supply company can realize the quick and accurate location of the fault point, and then send personnel to the scene to check immediately. In addition, after selecting the communication module, the integrated fault isolator can automatically report the fault information and line status information through the information channel which is wireless or wired.

\section{User load monitor}

If the communication module is configured, the fault isolator can possess the protection function. the remote terminal can realize the 'three remote' function and integrated management system can be established to monitor the user load in real time.

\subsection{Application of fault location system}

The fault location system is mainly composed of front-end wireless sensor, cloud network and background cloud data processing center. By using the method of real-time monitoring, the situation of power transmission, grounding fault and short circuit fault can be found in time. Then with the help of the fault indicator, the collected information data of the instantaneous fault waveform and 
features can be sent to the gathering unit in time. The gathering unit then sends the data to the cloud server via a wireless private network. The system software collects data analysis, information and waveform information in case of fault, and then sends it to the monitoring center. Through the fault software, the notification of alarm display, data analysis, cloud computing, report query and fault retrieval statistics can be realized, which can guide the maintenance personnel to locate the fault point quickly and accurately, and then achieve the improvement of work efficiency and the reduction of work intensity. At the same time, the fault information can be sent to the mobile phone of the head of the power supply production department and the line maintenance personnel by short message.

\subsection{Application of background platform system}

The cloud computing system of the background monitoring center formulates the unified interface standard of distribution network equipment monitoring and communicates directly with the fault isolation and location device. Fault isolation and area protection are carried out in front-end field, and field fault monitoring data are obtained by background system. The algorithms of short circuit grounding fault monitoring, real-time load monitoring, background fault topology distribution map and so on provide auxiliary analysis methods for field monitoring personnel. At the same time, the platform will build a knowledge base model. The platform effectively manages monitoring data and blackout test data by means of information technology, realizes the analysis and diagnosis of specialized monitoring data, and makes it a professional tool for monitoring and analysis of operation and maintenance units.

\subsection{Application of grounding short circuit technology}

\section{Short circuit Fault Judgment Method}

The electromagnetic induction method is used to judge the fault based on the short circuit characteristics. In the short circuit monitoring part of the fault detector, the sudden change of line current is detected by electromagnetic induction. In addition, the power failure and fault duration are monitored. The load current is automatically adapted and based on the fixed value of substation line protection, which can effectively avoid mal-operation.

Four conditions should be satisfied in the abrupt judgment of permanent short circuit using load current. First of all, it should be ensured that the normal operation time of the line is more than 30 seconds. Secondly, the abrupt current in the line is larger than 100A, or larger than the set detection parameters of the short circuit fault. Thirdly, the duration of the large current should be less than 10 seconds, i.e., $0.02 \mathrm{~s} \leq \Delta \mathrm{T} \leq 10 \mathrm{~s}$, where $\Delta \mathrm{T}$ is the time of current abruption. Finally, after 10 seconds the circuit should be blackout. Only if the above four conditions are satisfied can the fault detector be used to judge the permanent or the instantaneous line short circuit fault in this position. In order to ensure that the indicator lamp of non-fault branch will not be mal-operated when reclosing, the outrage can be utilized. After the time of electric field and current is more than 30 seconds, the locking can be lifted, and the short circuit current signal and the fault indicating short circuit can be responded to.

\section{Design of Ground Fault Judgment Scheme}

If there is a single-phase grounding fault in the line, there will be many complicated transient conditions due to the different grounding conditions and neutral grounding methods, such as the fault of discharge by line-to-ground distribution capacitor, or the increase of higher harmonics and the decrease of the ground voltage of the grounding line, etc. The details are shown in the Table. 1 below.

Table.1 Fault feature table of different neutral point grounding meyhods

\begin{tabular}{|c|c|c|c|}
\hline & $\begin{array}{c}\text { Neutral } \\
\text { point } \\
\text { ungrounded }\end{array}$ & $\begin{array}{c}\text { Neutral } \\
\text { point } \\
\text { solidly } \\
\text { grounded }\end{array}$ & $\begin{array}{c}\text { Neutral } \\
\text { point } \\
\text { grounded } \\
\text { via } \\
\text { suppression } \\
\text { coil } \\
\end{array}$ \\
\hline $\begin{array}{l}\text { Grounding } \\
\text { Current }\end{array}$ & $\begin{array}{l}\text { Transient } \\
\text { capacitance } \\
\text { current }\end{array}$ & $\begin{array}{c}\text { Massive } \\
\text { current at } \\
\text { fault phase } \\
\text { and flowing } \\
\text { into fault } \\
\text { point }\end{array}$ & $\begin{array}{l}\text { Grounding } \\
\text { current can } \\
\text { reduce to } \\
\text { very small }\end{array}$ \\
\hline $\begin{array}{l}\text { Neutral } \\
\text { Point } \\
\text { Voltage }\end{array}$ & $\begin{array}{l}\text { Increase to } \\
\text { phase } \\
\text { voltage }\end{array}$ & $\begin{array}{l}\text { Both fault } \\
\text { phase and } \\
\text { neutral } \\
\text { point } \\
\text { voltage is } 0\end{array}$ & $\begin{array}{l}\text { Increase to } \\
\text { phase } \\
\text { voltage }\end{array}$ \\
\hline $\begin{array}{c}\text { Non-fault } \\
\text { Phase } \\
\text { Voltage }\end{array}$ & $\begin{array}{l}\text { Increase to } \\
\text { line voltage }\end{array}$ & No change & $\begin{array}{l}\text { Increase to } \\
\text { line voltage }\end{array}$ \\
\hline $\begin{array}{c}\text { Line } \\
\text { Voltage }\end{array}$ & $\begin{array}{c}\text { Same as } \\
\text { normal }\end{array}$ & $\begin{array}{l}\text { Fault } \\
\text { related line } \\
\text { voltage } \\
\text { reduce to } \\
\text { phase } \\
\text { voltage }\end{array}$ & $\begin{array}{c}\text { Same as } \\
\text { normal }\end{array}$ \\
\hline
\end{tabular}

(Note: The above are the fault characteristics of each neutral grounding system when the conventional grounding fault occurs)

In view of the above fault characteristics, the project uses real-time acquisition of three-phase line on-line current, line to ground electric field, frozen fault threephase transient current, three-phase wireless synchronous calculation of zero-sequence current and other methods to conduct comprehensive ground fault judgment.

\section{Conclusions}

Combined with the actual situation of the project, this paper comprehensively analyzes and summarizes the existing distribution monitoring technology. By analyzing the principle of automatic fault diagnosis isolation device and system integration module, the application of automatic fault diagnosis isolation device system is discussed. It can be seen that the automatic fault diagnosis and isolation device of distribution line has the advantage of monitoring the load current and voltage of the line in 
real time and providing effective guarantee for the line operation. It can locate the fault point quickly and avoid the expansion of the accident, which can greatly reduce the labor intensity of the staff and improve the working efficiency. It is worth further studying and popularizing.

\section{References}

1. Z. C. Ma, L. Huo, P. Liu. Research on fast positioning and isolation device for $10 \mathrm{kV}$ distribution line fault $[\mathrm{J}]$. Global Market, 2017, no.3.

2. Y. Chen, J. Yuan. Fault analysis and intelligent device diagnosis of distribution line $[\mathrm{J}]$. Technology Wind, 2017, pp.146

3. Q. Zhao. Application of Fault Automatic Location and Isolation Technology of Distribution Line in 10 $\mathrm{kV}$ Distribution Network[J]. Telecom Power Technology, 2019, 36(8)

4. Z. L. Xu. Analysis of automatic fault location and isolation technology of distribution line in $10 \mathrm{kV}$ distribution network[J]. Science and Technology \& Innovation, 2019, 20.

5. Thukaram, D., H.P.Khincha, L.Jenkins, et al. A Three Phase Fault Detection Algorithm For Radial Distribution Networks[C]//Proceedings of IEEE Region 10 Conference on Computers Communications Control and Power Engineering, 2012, 2: 1242-1248.

6. Mora-Flores, J.Cormane-Angarita, Ordonez-Plata. Kmeans Algorithm and Mixture Distributions for Locating Faults in Power Systems[J]. Electric Power Systems Research, 2009, 79: 714-721.

7. T. Johns, P.Agrawal. New Approach to Power Line Protection Based on the Detection of Fault Induced High Frequency Signals. IEEE Transactions on Power Delivery, 2006, 137(4): 307-314.

8. Gilany M, Ibrahim D K. Traveling wave based faultlocation scheme for multi end-aged underground cable system[J]. IEEE Transactions on Power Delivery, 2007, 22(1): 82-89.

9. LI Rui-feng, ZENG Xiang-jun, LIU Hui, et al. The application of precision clock synchronization technology based on PTP (IEEE1588) in traveling wave fault location system[C]//The International Conference on Advanced Power System Automation and Protection, 2011, 5: 1631-1635. 